\title{
Application of an Elongated Kelvin Model to Space Shuttle Foams
}

\author{
Roy M. Sullivan, ${ }^{*}$ Louis J. Ghosn, ${ }^{\dagger}$ and Bradley A. Lerch $^{\ddagger}$ \\ NASA John H. Glenn Research Center at Lewis Field, Cleveland, Ohio 44135
}

\author{
DOI: $10.2514 / 1.37555$
}

\begin{abstract}
The space shuttle foams are rigid closed-cell polyurethane foams. The two foams used most extensively on the space shuttle external tank are BX-265 and NCFI24-124. Because of the foaming and rising process, the foam microstructures are elongated in the rise direction. As a result, these two foams exhibit a nonisotropic mechanical behavior. A detailed microstructural characterization of the two foams is presented. Key features of the foam cells are described and the average cell dimensions in the two foams are summarized. Experimental studies are also conducted to measure the room temperature mechanical response of the two foams in the two principal material directions (parallel to the rise and perpendicular to the rise). The measured elastic modulus, proportional limit stress, ultimate tensile strength, and Poisson's ratios are reported. The generalized elongated Kelvin foam model previously developed by the authors is reviewed and the equations which result from this model are summarized. Using the measured microstructural dimensions and the measured stiffness ratio, the foam tensile strength ratio and Poisson's ratios are predicted for both foams and are compared with the experimental data. The predicted tensile strength ratio is in close agreement with the measured strength ratio for both BX-265 and NCFI24-124. The comparison between the predicted Poisson's ratios and the measured values is not as favorable.
\end{abstract}

\begin{tabular}{|c|c|c|}
\hline \multicolumn{3}{|r|}{ Nomenclature } \\
\hline$A$ & $=$ & edge cross-sectional area, $\mu \mathrm{m}^{2}$ \\
\hline$b$ & $=$ & cell edge length, $\mu \mathrm{m}$ \\
\hline$C_{1}$ & $=$ & $\begin{array}{l}\text { nondimensional constant relating the edge cross- } \\
\text { sectional area to } r^{2}\end{array}$ \\
\hline$C_{2}$ & $=$ & $\begin{array}{l}\text { nondimensional constant relating the edge momen } \\
\text { of inertia to } A \cdot r^{2}\end{array}$ \\
\hline$C_{3}$ & $=$ & $\begin{array}{l}\text { nondimensional constant relating the edge section } \\
\text { modulus to } A \cdot r\end{array}$ \\
\hline$D$ & $=$ & unit cell width, $\mu \mathrm{m}$ \\
\hline$E$ & $=$ & solid material Young's modulus, MPa \\
\hline$E_{x}$ & $=$ & $\begin{array}{l}\text { perpendicular-to-rise direction foam Young's } \\
\text { modulus (along the } x \text { direction), } \mathrm{MPa}\end{array}$ \\
\hline$E_{y}$ & $=$ & $\begin{array}{l}\text { perpendicular-to-rise direction foam Young's } \\
\text { modulus (along the } y \text { direction), } \mathrm{MPa}\end{array}$ \\
\hline$E_{z}$ & $=$ & $\begin{array}{l}\text { foam rise direction Young's modulus } \\
\text { (along the } z \text { direction), MPa }\end{array}$ \\
\hline$G$ & $=$ & solid material shear modulus, $\mathrm{MPa}$ \\
\hline$H$ & $=$ & unit cell height, $\mu \mathrm{m}$ \\
\hline$I$ & $=$ & edge moment of inertia, $\mu \mathrm{m}^{4}$ \\
\hline
\end{tabular}

Presented as Paper 1786 at the 49th AIAA/ASME/ASCE/AHS/ASC Structures, Structural Dynamics, and Materials Conference, 16th AIAA/ ASME/AHS Adaptive Structures Conference, 10th AIAA Non-Deterministic Approaches Conference, 9th AIAA Gossamer Spacecraft Forum, 4th AIAA Multidisciplinary Design Optimization Specialist Conference, Renaissance Schaumburg Hotel and Convention Center, Schaumburg, IL, 7-10 April 2008; received 14 March 2008; revision received 15 July 2008; accepted for publication 13 August 2008. Copyright $(\odot) 2008$ by the American Institute of Aeronautics and Astronautics, Inc. The U.S. Government has a royalty-free license to exercise all rights under the copyright claimed herein for Governmental purposes. All other rights are reserved by the copyright owner. Copies of this paper may be made for personal or internal use, on condition that the copier pay the $\$ 10.00$ per-copy fee to the Copyright Clearance Center, Inc., 222 Rosewood Drive, Danvers, MA 01923; include the code 0022-4650/09 \$10.00 in correspondence with the CCC.

${ }^{*}$ Material Research Engineer, Structures and Materials Division, Mechanics and Life Prediction Branch, 21000 Brookpark Road, Mail Stop 49-7. AIAA Member.

'Principal Engineer, Structures and Materials Division, Ohio Aerospace Institute, 21000 Brookpark Road, Mail Stop 49-7.

${ }^{\ddagger}$ Material Research Engineer, Structures and Materials Division, Mechanics and Life Prediction Branch, 21000 Brookpark Road, Mail Stop 49-7.
$I_{x}^{L_{y}} \quad=$ moment of inertia of $L_{y}$ edges about the neutral axis parallel to the unit cell $x$ axis, $\mu \mathrm{m}^{4}$
$I_{y}^{L_{x}} \quad=$ moment of inertia of $L_{x}$ edges about the neutral axis parallel to the unit cell $y$ axis, $\mu \mathrm{m}^{4}$
$=$ moment of inertia of $b$-length edges about the neutral axis parallel to the unit cell $z$ axis, $\mu \mathrm{m}^{4}$
$=$ edge torsional constant, $\mu \mathrm{m}^{4}$
$=$ cell edge length, $\mu \mathrm{m}$
$=L$-length edges that bend about the neutral axis parallel to the unit cell $y$ axis, $\mu \mathrm{m}$
$=L$-length edges that bend about the neutral axis parallel to the unit cell $x$ axis, $\mu \mathrm{m}$
$=$ number of measurements
$=$ nondimensional cell shape parameter, $Q=b /(L \cos \theta)$
$=$ nondimensional cell shape parameter, $\tilde{Q}=2+\sqrt{2} Q=2+(\sqrt{2} b) /(L \cos \theta)$
$=$ average cell aspect ratio, $H / D$
$R$
$R_{E}$
$=$ foam stiffness ratio, $E_{z} / E_{x}=E_{z} / E_{y}$
$=$ foam strength ratio, $\sigma_{z z}^{s} / \sigma_{x x}^{s}=\sigma_{z z}^{s} / \sigma_{y y}^{s}$
$=$ edge cross section characteristic dimension (equal to the cross section radius for a circular or Plateau border cross section), $\mu \mathrm{m}$
$S \quad=$ section modulus, $\mu \mathrm{m}^{3}$
$S_{x}^{L_{y}} \quad=$ section modulus of $L_{y}$ edges about the neutral axis
$\begin{array}{ll} & \text { parallel to the unit cell } x \text { axis, } \mu \mathrm{m}^{3} \\ S_{y} L_{x} & =\end{array}$ parallel to the unit cell $y$ axis, $\mu \mathrm{m}^{3}$
$S_{z}^{b}=$ section modulus of $b$-length edges about the neutral axis parallel to the unit cell $z$ axis, $\mu \mathrm{m}^{3}$
$T \quad=$ nondimensional cell shape parameter, $\sqrt{16+\tilde{Q}^{2} R^{2}}=\sqrt{16+(2+\sqrt{2} Q) R^{2}}$
$x$
$=$ Cartesian coordinate perpendicular to the rise direction and normal to the $y$ direction
$=$ Cartesian coordinate perpendicular to the rise direction and normal to the $x$ direction
$=$ Cartesian coordinate along the rise direction
$z \quad=$ foam relative density
$\gamma \quad=$ inclination angle, deg
$v_{x y}=v_{y x}$
$=$ foam Poisson's ratio in the plane perpendicular to the rise direction




\begin{tabular}{|c|c|c|}
\hline$v_{z x}$ & $=$ & $\begin{array}{l}\text { foam Poisson's ratio for contraction in the } x \\
\text { direction due to tensile loading in the rise direction }\end{array}$ \\
\hline$v_{z y}$ & $=$ & $\begin{array}{l}\text { foam Poisson's ratio for contraction in the } y \\
\text { direction due to tensile loading in the rise direction }\end{array}$ \\
\hline$v_{x z}$ & $=$ & $\begin{array}{l}\text { foam Poisson's ratio for contraction rise direction } \\
\text { due to tensile loading in the } x \text { direction }\end{array}$ \\
\hline$v_{y z}$ & $=$ & $\begin{array}{l}\text { foam Poisson's ratio for contraction rise direction } \\
\text { due to tensile loading in the } y \text { direction }\end{array}$ \\
\hline$\sigma^{s}$ & $=$ & solid material intrinsic strength, $\mathrm{kPa}$ \\
\hline$\sigma_{x x}^{s}$ & $=$ & $\begin{array}{l}\text { foam perpendicular-to-rise direction strength (along } \\
\text { the } x \text { direction), } \mathrm{kPa}\end{array}$ \\
\hline$\sigma_{y y}^{s}$ & $=$ & $\begin{array}{l}\text { foam perpendicular-to-rise direction strength (along } \\
\text { the } y \text { direction), } \mathrm{kPa}\end{array}$ \\
\hline$\sigma_{z z}^{s}$ & $=$ & $\begin{array}{l}\text { foam rise direction strength (along the } z \text { direction), } \\
\mathrm{kPa}\end{array}$ \\
\hline
\end{tabular}

\section{Introduction}

$\mathbf{S}$ PRAY-ON foam insulation is applied to the exterior of the space shuttle's external tank to limit propellant boil off and to prevent ice formation. As a result of the catastrophic loss of the Space Shuttle Columbia, numerous studies have been conducted which aim to reduce the likelihood and the severity of foam shedding events during the Shuttle's ascent to space. Some of these studies have focused on understanding the mechanisms that cause foam fracture and debris liberation. This requires a thorough understanding of the foam mechanical response behavior and the mechanics of foam fracture.

The space shuttle foams are rigid polyurethane, closed-cell foams. The two foams used most extensively on the space shuttle external tank are BX-265 and NCFI24-124. NCFI24-124 is applied with an automated spray process and covers the majority of the exterior surface of the tank. The BX-265 is generally sprayed manually with a handheld spray gun. The microstructure of BX-265 and NCFI24-124 foams is illustrated in the photomicrographs shown in Fig. 1. Notice that both foams possess a distribution of cell sizes and shapes and that the cells in both foams tend to have an elongated shape. Because of the foaming and rising process, the foam microstructure is elongated in the rise direction. The elongated microstructure causes the foam mechanical response and strength behavior to be nonisotropic. Wright and Lerch [1] measured the average cell dimensions and average number of faces per cell in both BX-265 and NCFI24-124 using a scanning electron microscope. They found that the average
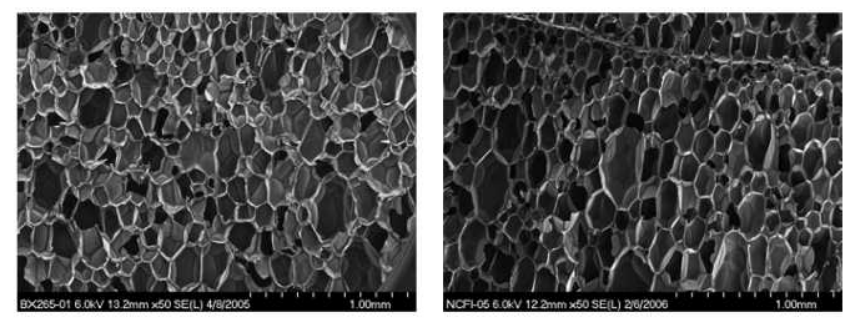

a) $\mathrm{BX}-265$

b) NCFI24-124

Fig. 1 Photomicrographs of a) BX-265 and b) NCFI24-124.

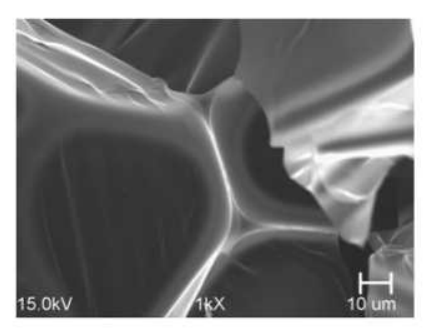

a) Cell faces and cell edges

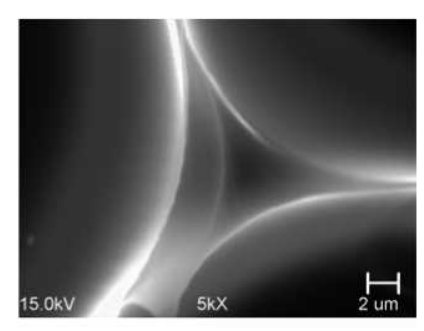

b) Edge cross section
Fig. 2 Photomicrographs showing a) the distinction between faces and edges and $b$ ) the cross section of an edge. number of faces per cell was 12.4 for BX-265 and 13.7 for NCFI24124.

The photomicrographs shown in Fig. 2 are higher resolution images of the foam microstructure, illustrating the features of a typical cell. The cells are formed by both faces and edges; the cell edges are formed by the intersection of multiple faces (see Fig. 2a). Figure $2 b$ is a close-up view of a cell edge cross section. The shape of the cell edge cross section resembles a three-cusp hypocycloid, otherwise known as a Plateau border cross section.

The photomicrograph in Fig. 1b shows a portion of a knit line in the upper half of the photo. A knit line is a higher density region that forms between successive spray layers. The cell dimensions are smaller and the cell edges and faces tend to be thicker in the knit lines, causing the knit lines to be a stronger and stiffer region of the microstructure.

Numerous studies [2-11] have attempted to develop equations which express the relation between the foam microstructure and the physical, mechanical, and strength properties of open- and closedcell foams. Many of these studies used a tetrakaidecahedron as the repeating unit cell. A tetrakaidecahedron is a 14-sided polyhedron possessing eight hexagonal and six quadrilateral sides and packs to fill space. The tetrakaidecahedron foam model is commonly referred to as the Kelvin foam model after Thomson [12]. Zhu et al. [5] and Warren and Kraynik [6] adopted an equiaxed tetrakaidecahedron to develop equations for the foam Young's modulus, shear modulus, and Poisson's ratio for isotropic, open-celled foams. They assumed that the mechanical behavior of open-celled foams could be simulated by treating the cell edges as structural elements possessing axial, bending, and torsional rigidity. The equations for the foam elastic constants were derived and written in terms of the cell edge length $L$, the edge cross-sectional area $A$, moment of inertia $I$, and torsional constant $J$, as well as the Young's modulus $E$, and shear modulus $G$ of the solid material.

To treat nonisotropic foams with a preferentially elongated microstructure, some researchers [7-10] adopted an elongated tetrakaidecahedron as their repeating unit cell. An elongated tetrakaidecahedron also packs to fill space and possesses eight hexagonal sides, two horizontal square sides, and four rhombicshaped vertical sides (see Fig. 3). The horizontal square faces have sides of length $b$, and the diamond faces have sides of length $L$. The hexagonal faces have four sides with length $L$ and two sides with length $b$. The inclination angle $\theta$ defines the orientation of the hexagonal faces with respect to the rise direction $z$, as well as the obtuse angle of the vertical diamond faces $2 \theta$.

Dement'ev and Tarakanov [7] used the elongated Kelvin model to derive equations for the elastic constants and compressive strengths, in the two principal material directions, for nonisotropic, open-celled foams. The equations for the elastic constants were derived by only considering the flexural deformation of the cell edges under the applied stresses. The compressive strength equations were obtained based on the critical (Euler) buckling load of the edges. Gong et al. $[8,9]$ also adopted an elongated Kelvin model and improved upon the fidelity of the previous models by using Plateau borders to represent the edge cross sections and by allowing the dimensions of the cross section to vary along the edge length. They also included the effects of shear deformation.

The size and shape of an elongated tetrakaidecahedron is uniquely defined by specifying the value of any three of the unit cell
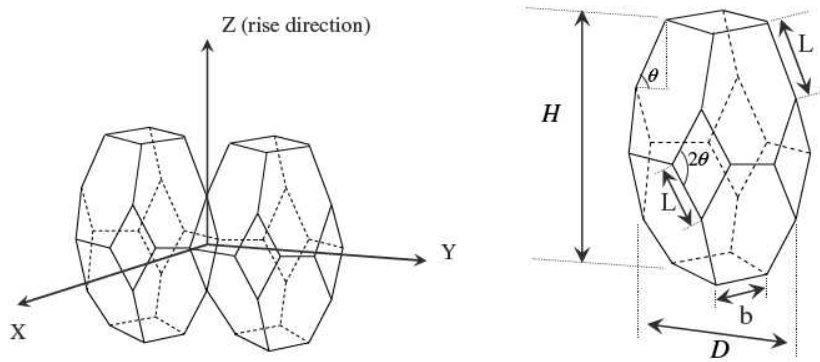

Fig. 3 Sketch of an elongated Kelvin foam model repeating unit cell. 
dimensions $H, D, b, L$, and $\theta$, because the cell height $H$ and cell width $D$ are related to $b, L$, and $\theta$ by

$$
H=4 L \sin \theta \text { and } D=2 L \cos \theta+\sqrt{2} b
$$

Thus, to apply the elongated Kelvin unit cell model to predict foam behavior, one must specify three foam microstructural dimensions to define the size and shape of the repeating unit cell, in addition to specifying the edge cross section shape and cross section dimensions.

Previous authors [7-10] have developed their equations by placing the restriction on the unit cell geometry that $b / L=\sqrt{2} \cos \theta$. This reduces the complexity of the derivations by reducing the number of independent dimensions. However, this restriction on the unit cell shape limits the generality of the model and could jeopardize its applicability to the widest range of foams.

Recently, Sullivan et al. [11] has developed equations for the elastic constants and tensile strengths for nonisotropic, open-celled foams using the most general description of an elongated Kelvin unit cell geometry. Using an approach similar to Zhu et al. [5], equations were derived and written as a function of the repeating unit cell dimensions $b, L$, and $\theta$, the modulus $E$, and strength $\sigma^{s}$ of the solid material and the edge cross section properties. Sullivan et al. [11] demonstrated the effect of this additional variation in the cell microstructure on the nonisotropic stiffness and strength behavior.

The objective of this paper is to apply the equations from Sullivan et al. [11] and attempt to model the elastic response and strength behavior of the two space shuttle external tank foams: BX-265 and NCFI24-124 foams. In applying the open-cell micromechanics model to simulate the mechanical and strength behavior of the closed-cell space shuttle foams, we must proceed under the assumption that the majority of the solid mass is concentrated in the cell edges and that the faces do not contribute significantly to the overall mechanical behavior of these foams.

In this paper, the micromechanics model from Sullivan et al. [11] is first reviewed. We then report on the results of some recent experimental studies that have been conducted on BX-265 and NCFI24-124 specimens. We report on our efforts to measure the average cell dimensions, the average mass density, and the tensile mechanical behavior of the two foams. In the mechanical testing, we measured the elastic modulus, the proportional limit stress, the ultimate strength, and the Poisson's ratios for the two foams in the principal material directions (parallel to the rise and perpendicular to the rise). The micromechanics model is applied to BX-265 and NCFI24-124 foams. Using some of the measured microstructural dimensions, the measured relative densities and the ratio of the measured elastic modulus in the rise direction to that in the perpendicular-to-rise direction, the remaining microstructural dimensions are predicted. We also predict the ratio of the tensile strengths in the two directions as well as the Poisson's ratios for both foams.

\section{Review of the General Micromechanics Model}

Sullivan et al. [11] derived the equations for the elastic constants and strengths in the principal material directions by treating the cell edges as structural members possessing both axial and flexural rigidity and by applying the minimum potential energy theorem to the unit cell (Fig. 3) deformation. They developed their equations using the simplifying assumption that the edge cross sections were constant along the length, and they wrote the equations for the elastic constants and strengths in terms of the unit cell dimensions $b, L$, and $\theta$, the modulus $E$, and strength $\sigma^{s}$ of the solid material and the edge cross section properties. The equations for the elastic constants and the tensile strengths are listed in the Appendix. Note that, due to symmetry of the unit cell, $E_{x}=E_{y}, v_{x z}=v_{y z}$, and $\sigma_{x x}^{s}=\sigma_{y y}^{s}$.

Under the action of the applied stresses $\sigma_{x x}, \sigma_{y y}$, and $\sigma_{z z}$, the edges will experience both axial and flexural deformations. The $L$-length edges will bend about the neutral axis, which is parallel to the unit cell $x$ or $y$ axis (see Fig. 3). Under $x$-direction loading, half the $L$-length edges will bend about the neutral axis, which is parallel to the unit cell $y$ axis. We will denote these edges as the $L_{x}$ edges, and we will denote the moment of inertia and section modulus for this flexural deformation as $I_{y}^{L_{x}}$ and $S_{y}^{L_{x}}$, respectively. Likewise, under $y$-direction loading, the other half of the $L$-length edges ( $L_{y}$ edges) will bend about the neutral axis, which is parallel to the unit cell $x$ axis. We will denote the section properties for this flexural deformation as $I_{x}^{L_{y}}$ and $S_{x}^{L_{y}}$. Under $z$-direction loading, both the $L_{x}$ edges and the $L_{y}$ edges will bend about their respective neutral axes.

Under a perpendicular-to-rise ( $x$ or $y$ ) direction loading, the $b$ length edges will bend about the neutral axis, which is parallel to the unit cell $z$ axis. The section properties for this flexural deformation may be denoted as $I_{z}^{b}$ and $S_{z}^{b}$. If the $L_{x}$ edges, the $L_{y}$ edges, and the $b$ length edges are all assumed to have the same cross section, then $I_{x}^{L_{y}}=I_{y}^{L_{x}}=I_{z}^{b}$ and $S_{x}^{L_{y}}=S_{y}^{L_{x}}=S_{z}^{b}$. The equations for the elastic constants and strengths in the Appendix are therefore written in terms of a single moment of inertia $I$ and section modulus $S$, where $I=I_{x}^{L_{y}}=I_{y}^{L_{x}}=I_{z}^{b}$ and $S=S_{x}^{L_{y}}=S_{y}^{L_{x}}=S_{z}^{b}$.

In addition, we note that, for any of the shapes which are typically used, the section properties are a function of a single cross section dimension. For example, the section properties, for a Plateau border cross section, are only a function of the cross section radius $r$, as seen in Fig. 4. We can thus specify all the cross section properties by specifying the value of the cross section radius. Note also that, if we assume a Plateau border cross section, the section modulus is $S=(60-11 \sqrt{3} \pi) r^{3} / 24$.

The tensile strength equations were derived under the assumption that foam failure initiated when the maximum stress in any cell edge was equal to the intrinsic strength of the solid (polyurethane) material $\sigma^{s}$. Under loading in the perpendicular-to-rise ( $x$ or $y$ ) direction, foam failure may occur due to failure of the $L$-length edges or the $b$-length edges. As a result, there are two equations listed in the Appendix for the perpendicular-to-rise direction foam strength. Equation (A3a) will always result in a lower estimate of the perpendicular-to-rise direction strength than Eq. (A3b) as long as the foam microstructure is such that $2 S \cos \theta+A L \sin \theta>\sqrt{2} S+A b / \sqrt{2}$. If this condition is met, the edges with length $L$ will fail at a lower applied stress than the edges of length $b$. We will proceed under the assumption that the perpendicular-to-rise direction strength is limited by failure of the $L$ length edges.

We would like to rewrite the equations in the Appendix in terms of the unit cell aspect ratio $R$ and the foam relative density $\gamma$, which is defined as the ratio of the foam density to the solid density. The aspect ratio of the unit cell is simply the ratio of the cell height to the cell width. Using Eq. (1), the aspect ratio may be written as

$$
R=\frac{H}{D}=\frac{4 L \sin \theta}{2 L \cos \theta+\sqrt{2} b}
$$

Using the unit cell shown in Fig. 3 and assuming that the edge crosssectional area $A$ is constant along the edge length, the foam relative density may be written as

$$
\gamma=\frac{2 A(2 L+b)}{L \sin \theta(2 L \cos \theta+\sqrt{2} b)^{2}}
$$

Sullivan et al. [11] also introduced a new shape parameter which they defined as $Q=b /(L \cos \theta)$. Using this expression, Eq. (2) can be rewritten as $R=4 \tan \theta /(2+\sqrt{2} Q)$, which leads to

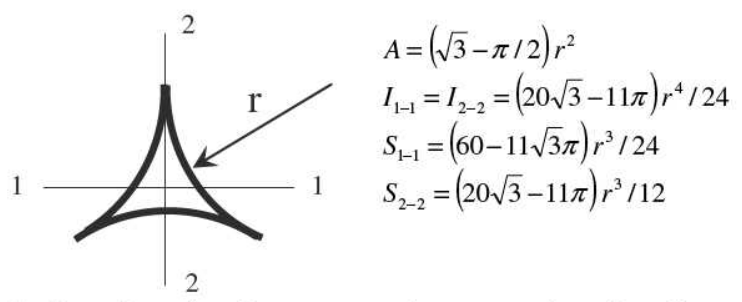

Fig. 4 Equations for the cross section properties of a three-cusp hypocycloid (Plateau border). 


$$
\cos \theta=\frac{4}{\sqrt{16+\tilde{Q}^{2} R^{2}}} \quad \sin \theta=\frac{\tilde{Q} R}{\sqrt{16+\tilde{Q}^{2} R^{2}}} \quad \frac{b}{L}=\frac{4 Q}{\sqrt{16+\tilde{Q}^{2} R^{2}}}
$$

where $\tilde{Q}=2+\sqrt{2} Q$. Notice that the restriction placed on the microstructure by previous authors [7-10] is such that $Q=\sqrt{2}$.

Using Eq. (2), Eq. (3) can be rearranged to obtain $A / L^{2}=8 \gamma \sin ^{3} \theta /(2+b / L) R^{2}$. Upon substituting Eqs. (4), this becomes

$$
\frac{A}{L^{2}}=\frac{4 \gamma \tilde{Q}^{3} R}{\left(16+\tilde{Q}^{2} R^{2}\right)\left(2 Q+\sqrt{16+\tilde{Q}^{2} R^{2}}\right)}
$$

Defining the constants $C_{1}, C_{2}$, and $C_{3}$ as $C_{1}=A / r^{2}, C_{2}=12 I / A r^{2}$, and $C_{3}=S / A r$, then $12 I / A L^{2}=\left(C_{2} / C_{1}\right) \cdot\left(A / L^{2}\right)$ and $S / A L=\left(C_{3} / \sqrt{C_{1}}\right) \sqrt{A / L^{2}}$. Substituting these relations along with Eqs. (4) and (5), the expressions in the Appendix for the elastic constants and strengths may be written in terms of the two shape parameters $R$ and $Q$ and the relative density $\gamma$ as

$$
\begin{gathered}
\frac{E_{z}}{E}=\frac{C_{2} \tilde{Q}^{5} R^{3} \gamma^{2} /(2 Q+T)^{2}}{4 C_{1} T+C_{2} \tilde{Q}^{5} R^{3} \gamma /\left(2 Q T+T^{2}\right)} \\
\frac{E_{x}}{E}=\frac{8 C_{2} \tilde{Q}^{5} R \gamma^{2} /(2 Q+T)^{2}}{\left(32 Q^{3}+\tilde{Q}^{2} R^{2} T\right) C_{1}+8 C_{2} \tilde{Q}^{3} R \gamma(8+Q T) /\left(2 Q T+T^{2}\right)}
\end{gathered}
$$

$$
v_{x y}=\frac{8 Q\left[4 C_{1} Q^{2}-C_{2} \tilde{Q}^{3} R \gamma /(2 Q+T)\right]}{\left(32 Q^{3}+\tilde{Q}^{2} R^{2} T\right) C_{1}+8 C_{2} \tilde{Q}^{3} R \gamma(8+Q T) /\left(2 Q T+T^{2}\right)}
$$

$$
v_{x z}=\frac{4 \tilde{Q}\left[T C_{1}-4 C_{2} \tilde{Q}^{3} R \gamma /\left(2 Q T+T^{2}\right)\right]}{\left(32 Q^{3}+\tilde{Q}^{2} R^{2} T\right) C_{1}+8 C_{2} \tilde{Q}^{3} R \gamma(8+Q T) /\left(2 Q T+T^{2}\right)}
$$

$$
\begin{gathered}
v_{z x}=\frac{\tilde{Q} R^{2}\left[C_{1}-4 C_{2} \tilde{Q}^{3} R \gamma /\left(2 Q T^{2}+T^{3}\right)\right]}{8 C_{1}+2 C_{2} \tilde{Q}^{5} R^{3} \gamma /\left(2 Q T^{2}+T^{3}\right)} \\
\frac{\sigma_{z z}^{s}}{\sigma^{s}}=\frac{C_{3} \tilde{Q}^{2.5} R^{1.5} \gamma^{1.5}}{(2 Q+T)^{1.5}\left[\sqrt{C_{1}}+C_{3} \tilde{Q}^{2.5} R^{1.5} \gamma^{0.5} /\left(2 Q T^{2}+T^{3}\right)^{0.5}\right]}(8 \mathrm{a}) \\
\frac{\sigma_{x x}^{s}}{\sigma^{s}} \\
=\frac{4 C_{3} \tilde{Q}^{2.5} R^{1.5} \gamma^{1.5}}{R(2 Q+T)^{1.5}\left[\sqrt{C_{1}} \tilde{Q} R+16 C_{3} \tilde{Q}^{1.5} R^{0.5} \gamma^{0.5} /\left(2 Q T^{2}+T^{3}\right)^{0.5}\right]}
\end{gathered}
$$

where $T=\sqrt{16+\tilde{Q}^{2} R^{2}}$

For a Plateau border cross section, the constants are $C_{1}=\sqrt{3}-\pi / 2, \quad C_{2}=(20 \sqrt{3}-11 \pi) /(2 \sqrt{3}-\pi)$, and $C_{3}=$ $(60-11 \sqrt{3} \pi) / 24(\sqrt{3}-\pi / 2)$.

The ratio of the stiffness in the rise direction to the stiffness in the perpendicular-to-rise direction can be written using Eqs. (6a) and (6b) as

$$
\begin{aligned}
R_{E} & =\frac{E_{z}}{E_{x}} \\
& =\frac{R^{2}}{8}\left[\frac{\left(32 Q^{3}+\tilde{Q}^{2} R^{2} T\right) C_{1}+8 C_{2} \tilde{Q}^{3} R \gamma(8+Q T) /\left(2 Q T+T^{2}\right)}{4 C_{1} T+C_{2} \tilde{Q}^{5} R^{3} \gamma /\left(2 Q T+T^{2}\right)}\right]
\end{aligned}
$$

The strength ratio follows from Eqs. (8a) and (8b) as

$$
R_{\sigma}=\frac{\sigma_{z z}^{s}}{\sigma_{x x}^{s}}=\frac{R}{4}\left[\frac{\sqrt{C_{1}} \tilde{Q} R+16 C_{3} \tilde{Q}^{1.5} R^{0.5} \gamma^{0.5} /\left(2 Q T^{2}+T^{3}\right)^{0.5}}{\sqrt{C_{1}}+C_{3} \tilde{Q}^{2.5} R^{1.5} \gamma^{0.5} /\left(2 Q T^{2}+T^{3}\right)^{0.5}}\right]
$$

\section{Experimental Studies}

The foam microstructure is dependent on many variables such as the geometric constraints imposed on the foam during the foaming process, the ambient temperature, and the ambient relative humidity. As such, there can be considerable variation in the microstructural features between two spray applications of the same foam formulation. To address this, samples for the microstructural measurements and mechanical properties were excised from the same block of foam. The mass density was measured on the tensile test specimens before performing the tensile tests. Thus, the density, the average microstructural dimensions, and the average tensile properties were measured from the same spray block. This eliminated any error associated with spray-to-spray variability.

Although the tensile specimens contain knit lines, the volume of the knit lines is only a small fraction of the specimen volume. We assume that the knit lines do not significantly affect the measured density, mechanical behavior, or strength of the specimens.

\section{A. Microstructural Characterization}

We measured the average cell height and average cell width in the BX-265 and NCFI24-124 spray blocks using a scanning electron microscope. We also measured the average cross section radius in the NCFI24-124 block. Unfortunately, we did not measure the average cross section radius in the $\mathrm{BX}-265$ block. The results of the microstructural measurements are summarized in Table 1, where the average values are listed for each of the foams along with the number of measurements taken $n$ to obtain each average value. Table 1 also lists the average aspect ratio $R=H / D$ for each foam. It appears that the cells in NCFI24-124 are more elongated than the cells in BX-265.

\section{B. Mechanical Property Testing and Density Measurements}

Specimens were excised from the BX-265 and NCFI24-124 blocks for mechanical property testing. The room temperature tensile stress-strain behavior of BX-265 and NCFI24-124 was measured using 50.8-mm-long (2.0 in.) parallelepiped specimens. The BX-265 specimens had a square cross section with the dimensions of $12.7 \times$ $12.7 \mathrm{~mm}(0.5 \times 0.5 \mathrm{in}$.) and the NCFI24-124 specimens had a rectangular cross section with the dimensions of $15.24 \times 10.16 \mathrm{~mm}$ $(0.6 \times 0.4 \mathrm{in}$.). To measure the material response in both material directions, specimens were cut so that the length dimension was coincident with the rise and perpendicular-to-rise directions.

The samples were glued to aluminum platens using epoxy and tested in a standard load frame. A stroke rate of $0.762 \mathrm{~mm}$ per minute was employed. A calibrated $222.4 \mathrm{~N}$ (50 lb) load cell was used to measure loads. Displacement was measured using both a linear variable displacement transducer (LVDT) associated with the load frame and a laser micrometer, which read the distance between two flags glued onto the sample. Both methods gave similar values. However, only the strains calculated from the LVDT will be used here, as the LVDT method resulted in a larger signal-to-noise ratio.

The tensile stress-strain curves are shown in Fig. 5. Figure 5a shows the BX-265 stress-strain response in the perpendicular-to-rise and rise direction, and Fig. 5b shows the NCFI24-124 results. Note that the strength and stiffness in the rise direction is considerably higher than in the perpendicular-to-rise direction for both materials. 
Table 1 Summary of microstructural measurements

\begin{tabular}{lccccccc}
\hline \hline & \multicolumn{2}{c}{ Cell height, $H$} & \multicolumn{2}{c}{ Cell width, $D$} & \multicolumn{2}{c}{ Edge cross section radius, $r$} & \multirow{2}{*}{ Aspect ratio $R=H / D$} \\
\cline { 1 - 5 } & Avg., $\mu \mathrm{m}$ & $n$ & Avg., $\mu \mathrm{m}$ & $n$ & Avg., $\mu \mathrm{m}$ & $n$ & \\
\hline BX-265 & 193 & 100 & 136 & 100 & NA $^{\mathrm{a}}$ & & 1.42 \\
NCFI24-124 & 248 & 100 & 142 & 100 & 26.0 & 27 & 1.75 \\
\hline \hline
\end{tabular}

a Data not available.

Table 2 Summary of tensile test results on BX-265

\begin{tabular}{|c|c|c|c|c|c|c|c|}
\hline & Specimen & Density, $\mathrm{g} / \mathrm{cm}^{3}$ & No. of knit lines & $E, \mathrm{MPa}$ & $\mathrm{PL}, \mathrm{kPa}$ & UTS, $\mathrm{kPa}$ & Fail location \\
\hline \multirow{7}{*}{ Perpendicular to rise } & L2 & 0.0405 & 3.0 & 8.21 & 227.5 & 359.8 & Gauge \\
\hline & L3 & 0.0368 & 2.0 & 6.82 & 165.4 & 301.9 & Gauge \\
\hline & $\mathrm{L}^{\mathrm{a}}$ & 0.0378 & 2.0 & 7.12 & 179.2 & 357.1 & Gauge \\
\hline & L5 & 0.0368 & 1.0 & 6.69 & 137.9 & 312.2 & Gauge \\
\hline & L6-1 ${ }^{\text {a }}$ & 0.0362 & 1.7 & 6.60 & 179.2 & 268.8 & Gauge \\
\hline & L7 & 0.0373 & 1.0 & 6.71 & 151.6 & 316.4 & Gauge \\
\hline & Avg. & & & 7.03 & 173.5 & 319.4 & \\
\hline \multirow{10}{*}{ Rise } & $\mathrm{T} 1$ & 0.0370 & 7.5 & 13.08 & - & - & Glue line \\
\hline & $\mathrm{T} 2$ & 0.0376 & 8.5 & 15.47 & 324.0 & 575.6 & Gauge \\
\hline & T3 & 0.0357 & 6.5 & 12.66 & 303.3 & 526.6 & Gauge \\
\hline & $\mathrm{T} 4$ & 0.0353 & 6.0 & 13.58 & 317.1 & - & Gauge at flag glue line \\
\hline & T5 & 0.0367 & 7.0 & 12.16 & - & - & Glue line \\
\hline & T6 & 0.0376 & 8.0 & 14.82 & 317.1 & 565.2 & Gauge \\
\hline & $\mathrm{T} 7^{\mathrm{a}}$ & 0.0337 & 3.0 & 12.33 & $\longrightarrow$ & - & Glue line \\
\hline & $\mathrm{T}^{\mathrm{a}}$ & 0.0370 & 4.0 & 14.14 & - & - - & Glue line \\
\hline & Avg. & & & 13.53 & 315.4 & 555.8 & \\
\hline & BX Avg. & 0.0369 & & & & & \\
\hline
\end{tabular}

aspecimen used for Poisson's ratio measurements.

Also, both materials exhibit an initial linear region followed by a nonlinear response leading up to failure. The nonlinear behavior of BX-265 is much more pronounced than NCFI24-124.

The initial modulus and proportional limit was estimated for each specimen from the stress-strain curves shown in Fig. 5. The estimated initial modulus, the estimated proportional limit (PL), and ultimate tensile strength (UTS) for the BX-265 and NCFI24-124 tensile tests are summarized in Tables 2 and 3, respectively. Also listed in Tables 2 and 3 are the density of the specimen, the number of knit lines in the specimen, and the location of fracture. The average density of the BX-265 and NCFI24-124 test specimens is $0.0369 \mathrm{~g} / \mathrm{cm}^{3}$ and $0.0373 \mathrm{~g} / \mathrm{cm}^{3}$, respectively. Assuming a density of $1.2 \mathrm{~g} / \mathrm{cm}^{3}$ for polyurethane, the relative density of both BX-265 and NCFI24-124 is approximately 0.031 .

A large number of the BX-265 rise direction specimens failed prematurely and, as a result, the strength values associated with these tests are not reported in Table 2. In all of these tests, the specimens failed at the glue line. In two of these tests (T1 and T5), the adhesive did not fully cure, which led to premature separation of the glue. All NCFI24-124 specimens failed in the gauge section.

In some of the tensile tests, the lateral strain in the specimen was also measured to determine the Poisson's ratios. The test specimens that were used to measure the Poisson's ratios are identified by footnote markers in Tables 2 and 3. The lateral strains were measured using a high-resolution noncontacting laser micrometer. Multiple lateral strain measurements were made within the same specimen at various locations within the gauge section. The Poisson's ratio for each specimen was calculated from the average lateral strain in the specimen divided by the measured longitudinal strain. The measured Poisson's ratios are listed in Table 4 along with the standard deviations.

The ratio of the modulus in the rise direction to that in the perpendicular-to-rise direction, as well as the ratio of the strengths in the two material directions, is indicative of the extent of elongation of

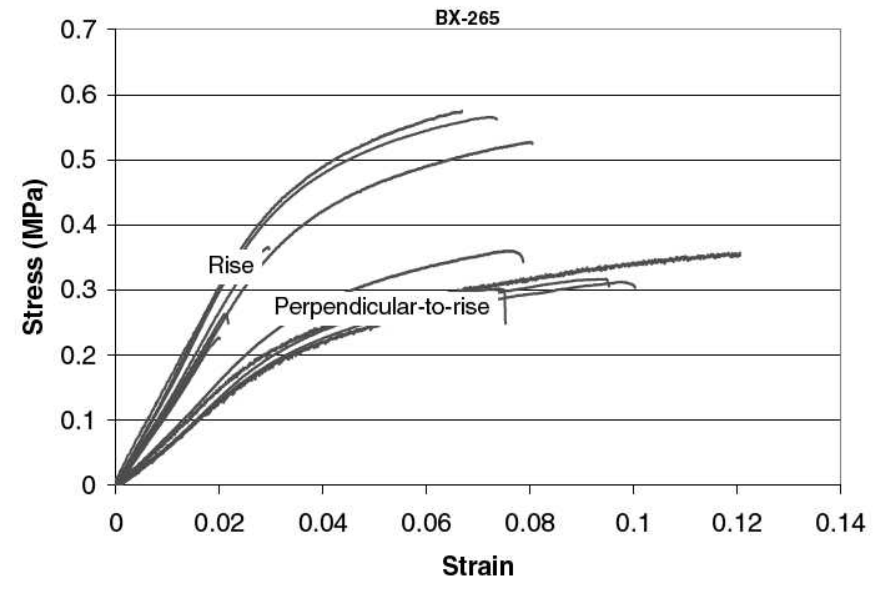

a) $\mathrm{BX}-265$

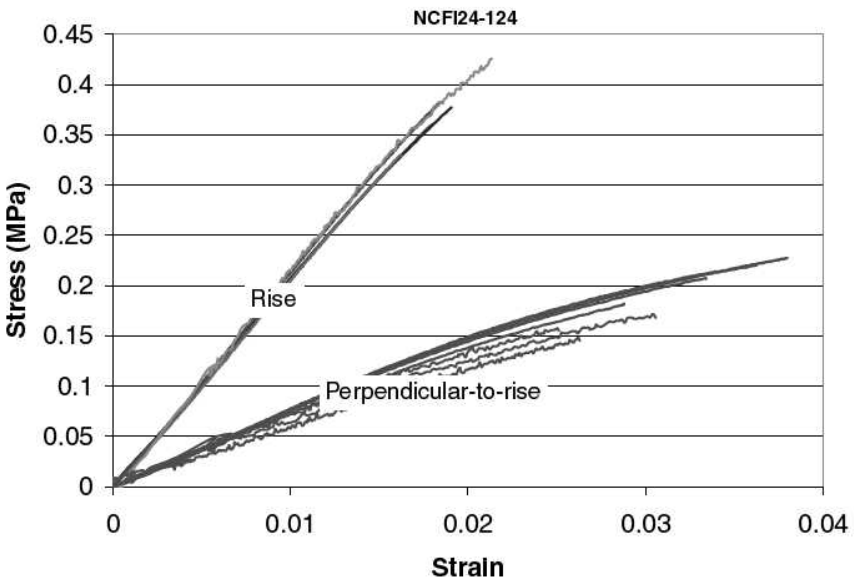

b) NCFI24-124

Fig. 5 Stress vs strain curves from tensile tests on a) BX-265 and b) NCFI24-124. 
Table 3 Summary of tensile test results on NCFI24-124

\begin{tabular}{|c|c|c|c|c|c|c|c|}
\hline & Specimen & Density, $\mathrm{g} / \mathrm{cm}^{3}$ & No. of knit lines & $E, \mathrm{MPa}$ & $\mathrm{PL}, \mathrm{kPa}$ & UTS, kPa & Fail location \\
\hline \multirow{8}{*}{ Perpendicular to rise } & L1 & 0.0367 & 2.1 & 7.09 & 131.0 & 182.7 & Gauge \\
\hline & L2 & 0.0393 & 2.2 & 7.53 & 103.4 & 227.5 & Gauge \\
\hline & L3 & 0.0385 & 2.6 & 7.49 & 110.3 & 208.2 & Gauge \\
\hline & $\mathrm{L} 4$ & 0.0382 & 2.8 & 7.73 & 117.2 & 220.6 & Gauge \\
\hline & $\mathrm{L}^{\mathrm{a}}$ & 0.0369 & 2.0 & 6.54 & 75.8 & 148.2 & Gauge \\
\hline & $\mathrm{L} 5-2^{\mathrm{a}}$ & 0.0369 & 2.0 & 6.56 & 117.2 & 157.8 & Gauge \\
\hline & L6- $4^{\mathrm{a}}$ & 0.0370 & 2.0 & 6.52 & 124.1 & 172.3 & Gauge \\
\hline & Avg. & & & 7.07 & 111.3 & 188.2 & \\
\hline \multirow{7}{*}{ Rise } & $\mathrm{T} 1$ & 0.0367 & 7.0 & 20.35 & 220.6 & 360.5 & Gauge \\
\hline & $\mathrm{T} 2$ & 0.0350 & 7.0 & 20.27 & 255.0 & 377.7 & Gauge \\
\hline & T3 & 0.0364 & 8.0 & 19.90 & 248.1 & 330.2 & Gauge \\
\hline & $\mathrm{T} 4$ & 0.0364 & 8.0 & 20.95 & 248.1 & 382.6 & Gauge \\
\hline & $\mathrm{T} 6^{\mathrm{a}}$ & 0.0390 & 4.0 & 22.52 & 282.6 & 426.0 & Gauge \\
\hline & Avg. & & & 20.80 & 250.9 & 375.4 & \\
\hline & NCFI Avg. & 0.0373 & & & & & \\
\hline
\end{tabular}

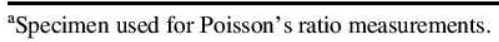

Table 4 Summary of measured Poisson's ratios

\begin{tabular}{lllll}
\hline \hline & & & \\
& & Specimen & Avg. measured Poisson's ratio & Standard deviation \\
\hline \multirow{3}{*}{ BX-265 } & $v_{x y}$ & L6-1 & 0.355 & 0.06 \\
& $v_{x z}$ & L4 & 0.273 & 0.0007 \\
& $v_{z x}$ & T7 & 0.536 & 0.29 \\
& $v_{z x}$ & T8 & 0.675 & 0.17 \\
NCFI24-124 & $v_{x y}$ & L6-4 & 0.382 & 0.14 \\
& $v_{x z}$ & L5 & 0.183 & 0.04 \\
& $v_{x z}$ & L5-2 & 0.160 & 0.03 \\
& $v_{z x}$ & T6 & 0.641 & 0.10 \\
\hline \hline
\end{tabular}

Table 5 Strength and stiffness ratios from tensile test results

\begin{tabular}{llll}
\hline \hline & Stiffness ratio & Proportional limit ratio & Ultimate tensile strength ratio \\
\hline BX-265 & 1.92 & 1.82 & 1.74 \\
NCFI24-124 & 2.94 & 2.25 & 1.99 \\
\hline \hline
\end{tabular}

the cells. The stiffness and strength ratios were calculated from the average strengths and average moduli listed in Tables 2 and 3 . These are listed in Table 5. Because the stiffness and strength ratios for BX265 are lower than for NCFI24-124, one would expect that an "average" cell in the NCFI24-124 specimens is more elongated than in the BX-265 specimens. This is consistent with the measured average aspect ratios listed in Table 1.

\section{Application of the Model to BX-265 and NCFI24-124}

Using Eq. (9), the stiffness ratio $R_{E}$ is plotted in Fig. 6 as a function of the aspect ratio $R$ for various values of the shape parameter $Q$. A Plateau border cross section and a relative density of 0.03 have been assumed. We have also plotted the measured stiffness ratio versus the average measured aspect ratio for BX-265 and NCFI24-124 as individual data points. These results indicate that $Q \approx 1$ for BX-265 and $Q<1$ for NCFI24-124.

The value of $Q$ was obtained for BX-265 and NCFI24-124 by solving Eq. (9) using the measured values for $\gamma, R$, and $R_{E}$. Once the value of $Q$ was obtained, the tensile strength ratio $R_{\sigma}$ for the two foams was calculated from Eq. (10). The results of these calculations are summarized in Table 6 . The measured strength ratios from Table 5 are also included for the sake of comparison. There is good agreement between the predicted strength ratios and the measure ratios for both BX-265 and NCFI24-124. In particular, the agreement between the predicted strength ratios and the measured proportional limit strength ratios are quite good.

The edge length $b$ was calculated from $b=D /(\sqrt{2}+2 / Q)$, which is obtained from the second expression in Eq. (1) and from the expression $Q=b /(L \cos \theta)$. The edge length $L$ was then determined from $b / L=4 Q / \sqrt{16+\tilde{Q}^{2} R^{2}}$, and the inclination angle $\theta$ was determined from $\cos \theta=b / Q L$. The predicted cross section radius $r$ was calculated from $A=C_{1} r^{2}=\gamma R D^{3} /(16 L+8 b)$, which is obtained from Eqs. (2) and (3).

Plateau Border Cross-sections; $\gamma=0.03$

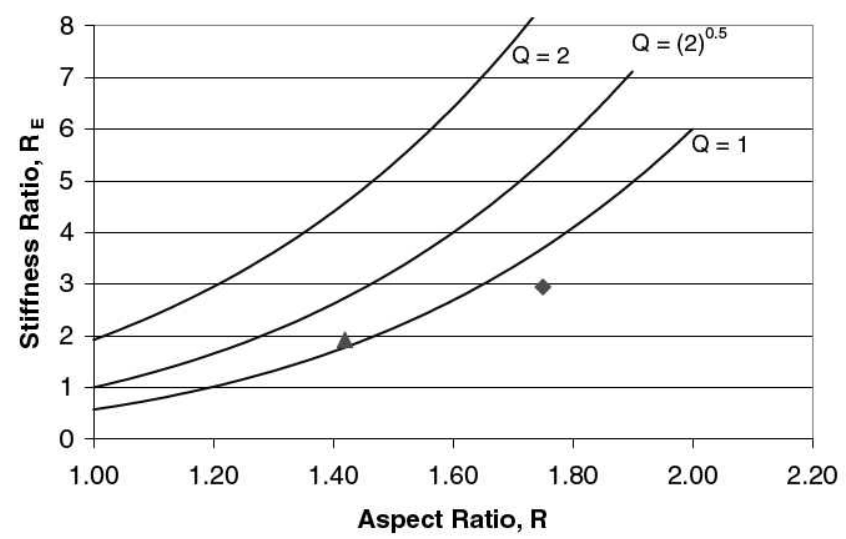

Fig. 6 Plot of the stiffness ratio $R_{E}$ vs cell aspect ratio $R$ for various values of the shape parameter $Q$, assuming Plateau border cross section and a relative density of $\gamma=\mathbf{0 . 0 3}$. Individual data points indicate the measured stiffness ratio and average measured aspect ratio for BX-265 and NCFI24-124. 
Table 6 Summary of analysis results and comparison to measurements

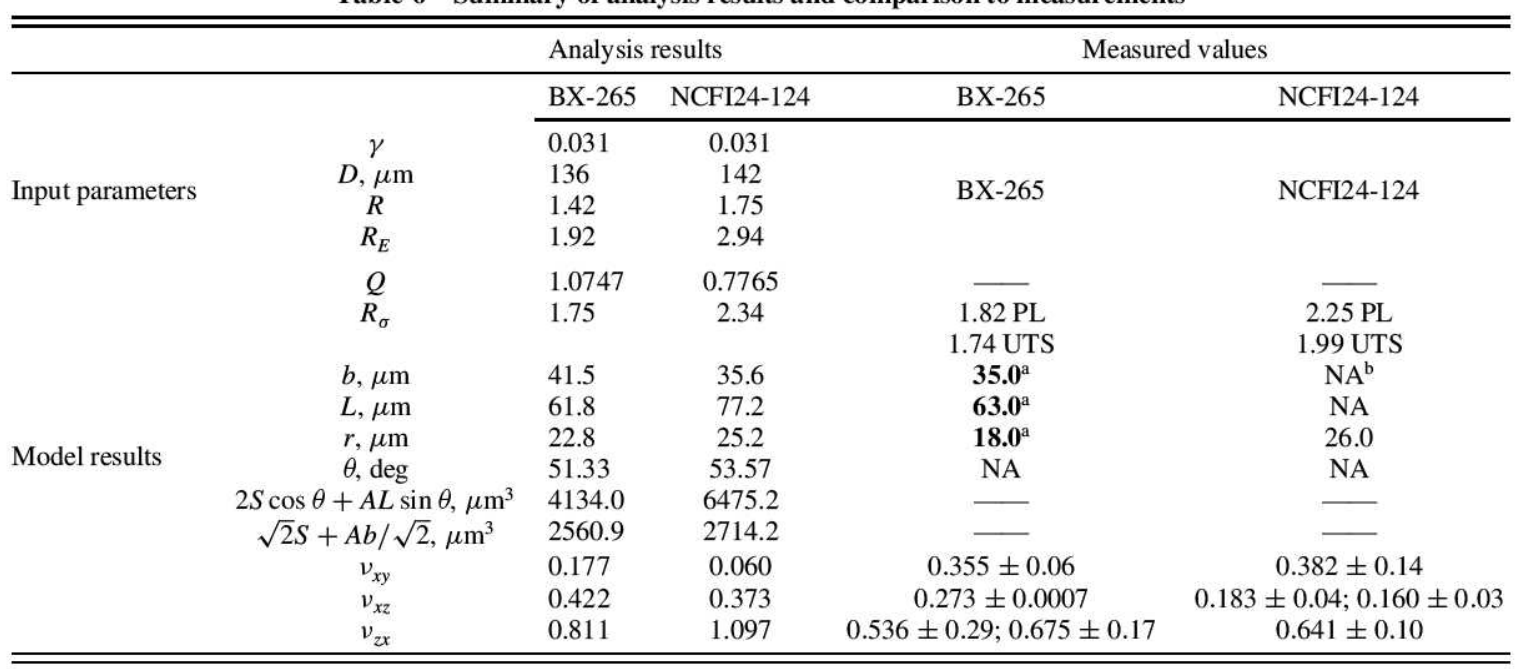

${ }^{\mathrm{a}}$ Measurements from another BX-265 spray block.

${ }^{\mathrm{b}}$ Data not available.

The predicted edge lengths and cross section radius for BX-265 and NCFI24-124 are listed in Table 6. The predicted edge cross section radius for NCFI24-124 compares very well with the measured average radius. Unfortunately, we do not currently have a measurement of the average edge lengths for this block of NCFI24124. We also do not have a measurement of the average edge lengths and average cross section radius for the specific block of BX-265 that was the subject of this study. We have obtained measurements of the average edge lengths and the average cross section radius in another block of BX-265 (see [13]). These measurements are shown in Table 6 in bold print. Note that the predicted values for the BX-265 edge lengths and cross section radius are close to the average values measured in the other block of BX-265. This provides us with further validation of the micromechanics model and its application to these types of foams.

Notice that the microstructure of both foams is such that $2 S \cos \theta+A L \sin \theta>\sqrt{2} S+A b / \sqrt{2}$. Thus, we are justified in using Eq. (A3a) to represent the perpendicular-to-rise direction strength in calculating the foam strength ratios.

The predicted values of the Poisson ratios $v_{x y}, v_{x z}$, and $v_{z x}$ are obtained from Eqs. (7a-7c), respectively. The predicted Poisson's ratios are listed in Table 6 along with the measured values from Table 4. Note that the predicted values for the Poisson's ratios do not agree with the measured values. For both foams, the predicted values for $v_{x y}$ are significantly lower than the measured values, and the predicted values for $v_{x z}$ and $v_{z x}$ are significantly higher than the measured values. In all cases except one ( $v_{z x}$ for BX-265), the predicted Poisson's ratios fall outside the range of values established by the measured average value plus or minus one standard deviation. The most likely reason for the poor correlation between the measured and predicted Poisson's ratios is that the open-celled micromechanics model neglects the effect of the cell faces and, in reality, the strain energy of the cell faces will effect the unit cell displacements in the direction normal to loading. We have recently performed finite element analysis of an elongated Kelvin unit cell with and without the cell faces [13]. The results of these finite element analyses [13] reveal that the effect of including the cell faces in the model is to increase the value of $v_{x y}$ and to decrease the values of $v_{x z}$ and $v_{z x}$.

\section{Conclusions}

Using the average measured cell dimensions $H$ and $D$, the average measured density and the measured stiffness ratio $R_{E}$, the equations which result from the general elongated Kelvin micromechanics model were successfully applied to predict the measured tensile strength ratio of BX-265 and NCFI24-124. These equations were also successful in predicting other microstructural dimensions, such as the edge lengths $b$ and $L$ and the edge cross section radius $r$. Consequently, we could expect that, given the average values for four of the microstructural dimensions, the model would predict the measured stiffness ratio and measured tensile strength ratio of these two foams. If the modulus and strength of the solid material is known, the model would then be capable of predicting the foam modulus and strength in the principal material directions. We thus conclude that the general elongated Kelvin foam model is a convenient tool for estimating the foam strength and stiffness from the average microstructural dimensions.

The comparison between the predicted Poisson's ratio and the measured values was not as favorable. The micromechanics model predicted a value for $v_{x y}$, which was less than the measured value for both BX-265 and NCFI24-124. Conversely, the model predicted values for $v_{x z}$ and $v_{z x}$ that were greater than what was measured for both foams. The reason for this discrepancy is due to the fact that the current micromechanics model neglects the cell faces and we believe the cell faces have a significant effect on the strains transverse to the loading direction.

Finally, we note that the microstructure of the BX-265 and NCFI24-124 foams appears to have a shape parameter $Q=1.075$ and $Q=0.777$, respectively. Thus, a model which restricts the foam microstructure to $Q=\sqrt{2}$ is inappropriate for BX-265 and NCFI24-124.

\section{Appendix: Summary of the Equations for the Elastic Constants and Strengths}

From Sullivan et al. [11], we summarize the equations for the elastic constants and strengths written in terms of $b, L, \theta, A, I$, and $S$.

Young's modulus:

$$
E_{x}=E_{y}=\frac{12 E I}{L \sin \theta\left[2 L^{3} \sin ^{2} \theta+b^{3}+(12 I / A)\left(2 L \cos ^{2} \theta+b\right)\right]}
$$

$$
E_{z}=\frac{24 E I \sin \theta}{L^{2}\left[\cos ^{2} \theta+\left(12 I / A L^{2}\right) \sin ^{2} \theta\right](\sqrt{2} L \cos \theta+b)^{2}}
$$

Poisson's ratio:

$$
v_{x y}=v_{y x}=\frac{b\left(A b^{2}-12 I\right)}{12 I\left(2 L \cos ^{2} \theta+b\right)+A\left(2 L^{3} \sin ^{2} \theta+b^{3}\right)}
$$




$$
\begin{aligned}
& v_{x z}=v_{y z}=\frac{\left(A L^{2}-12 I\right)(2 L \cos \theta+\sqrt{2} b) \cos \theta}{2\left[12 I\left(2 L \cos ^{2} \theta+b\right)+A\left(2 L^{3} \sin ^{2} \theta+b^{3}\right)\right]} \\
& v_{z x}=v_{z y}=\frac{\sqrt{2} L\left(A L^{2}-12 I\right) \cos \theta \sin ^{2} \theta}{\left(12 I \sin ^{2} \theta+A L^{2} \cos ^{2} \theta\right)(\sqrt{2} L \cos \theta+b)}
\end{aligned}
$$

Strength:

$x$ or $y$ direction (failure of $L$-length members):

$\sigma_{x x}^{s}=\sigma_{y y}^{s}=\frac{\sigma^{s}}{\left[(L \cos \theta \sin \theta / A)+\left(L^{2} \sin ^{2} \theta / 2 S\right)\right](2 L \cos \theta+\sqrt{2} b)}$

$x$ or $y$ direction (failure of $b$-length members):

$$
\sigma_{x x}^{s}=\sigma_{y y}^{s}=\frac{\sqrt{2} \sigma^{s}}{[(L \sin \theta / A)+(L b \sin \theta / 2 S)](2 L \cos \theta+\sqrt{2} b)}
$$

$z$ direction:

$$
\sigma_{z z}^{s}=\frac{\sigma^{s}}{[(\sin \theta / 2 A)+(L \cos \theta / 4 S)](\sqrt{2} L \cos \theta+b)^{2}}
$$

\section{Acknowledgment}

The authors are grateful for funding from the External Tank Project under NASA's Space Shuttle Program.

\section{References}

[1] Wright, L. S., and Lerch, B. A., "Characterization of Space Shuttle Insulative Materials," NASA TM-2005-213596, April 2005.

[2] Gent, A. N., and Thomas, A. G., "The Deformation of Foamed Elastic Materials," Journal of Applied Polymer Science, Vol. 1, No. 1, 1959, pp. 107-113.

doi:10.1002/app.1959.070010117

[3] Huber, A. T., and Gibson, L. J., "Anisotropy of Foams," Journal of
Materials Science, Vol. 23, No. 8, 1988, pp. 3031-3040. doi:10.1007/BF00547486

[4] Gibson, L. J., and Ashby, M. F., Cellular Solids: Structure and Properties, 2nd ed., Cambridge Univ. Press, Cambridge, England, UK, 1997.

[5] Zhu, H. X., Knott, J. F., and Mills, N. J., "Analysis of the Elastic Properties of Open-Cell Foams with Tetrakaidecahedral Cells," Journal of the Mechanics and Physics of Solids, Vol. 45, No. 3, 1997, pp. 319-343. doi:10.1016/S0022-5096(96)00090-7

[6] Warren, W. E., and Kraynik, A. M., "Linear Elastic Behavior of a LowDensity Kelvin Foam with Open Cells," Journal of Applied Mechanics, Vol. 64, No. 4, 1997, pp. 787-794. doi: $10.1115 / 1.2788983$

[7] Dement'ev, A. G., and Tarakanov, O. G., "Model Analysis of the Cellular Structure of Plastic Foams of the Polyurethane Type," Mekhanika Polimerov, Vol. 5, Sept.-Oct. 1970, pp. 859-865; also in Polymer Mechanics, Vol. 6, No. 5, 1970, pp. 744-749 (in English). doi:10.1007/BF00856206

[8] Gong, L., Kyriakides, S., and Jang, W. Y., "Compressive Response of Open-Celled Foams, Part I: Morphology and Elastic Properties," International Journal of Solids and Structures, Vol. 42, Nos. 5-6, 2005, pp. $1355-1379$. doi:10.1016/j.ijsolstr.2004.07.023

[9] Gong, L., Kyriakides, S., and Triantafyllidis, N., "On the Stability of Kelvin Cell Foams Under Compressive Loads," Journal of the Mechanics and Physics of Solids, Vol. 53, No. 4, 2005, pp. 771-794. doi:10.1016/j.jmps.2004.10.007

[10] Ridha, M., Shim, V. P. W., and Yang, L. M., "An Elongated Tetrakaidecahedral Cell Model for Fracture in Rigid Polyurethane Foam," Key Engineering Materials, Vols. 306-308, Pt. 1, 2006, pp. 43 48, http://www.scientific.net.

[11] Sullivan, R. M., Ghosn, L. J., and Lerch, B. A., "A General Tetrakaidecahedron Model for Open-Celled Foams," International Journal of Solids and Structures, Vol. 45, No. 6, 2008, pp. 1754-1765. doi:10.1016/j.ijsolstr.2007.10.028

[12] Thomson, W., Lord Kelvin, "On the Division of Space with Minimum Partitional Area," Philosophical Magazine, Vol. 24, No. 151, 1887, pp. 503-514, http://zapatopi.net/kelvin/papers/on_the_division_of_space.html.

[13] Sullivan, R. M., Ghosn, L. J., Lerch, B. A., and Baker, E. H., "An Elongated Tetrakaidecahedron Micro-Mechanics Model for Space Shuttle External Tank Foams," NASA TP-2008-215137, 2008.

L. Peterson Associate Editor 